\title{
Tuning Nonlinear Optical Behavior by Incorporation of the Chalcogenophene into Twistacenes
}

Yanbing Han, ${ }^{\dagger}$ Jinchong Xiao, ${ }^{*}$, Xingzhi Wu, ${ }^{\S}$ Wenfa Zhou, ' Lei Shen, ${ }^{\|}$Jian Zhang, + Y Yuxiao

Wang, ${ }^{\dagger}$ Xueru Zhang, ${ }^{\dagger}$ Hongwei Hou ${ }^{*}, \perp$ and Yinglin Song $*, \dagger$

'Department of Physics, Harbin Institute of Technology, Harbin 150001, P. R. China

${ }^{+}$College of Chemistry and Environmental Science, Key Laboratory of Chemical Biology of Hebei Province, Hebei University, Baoding 071002, P. R. China

§iangsu Key Laboratory of Micro and Nano Heat Fluid Flow Technology and Energy Applicati on, School of Mathematics and Physics, Suzhou University of Science and Technology, Suzhou 2 15009, China

'School of Physical Science and Technology, Soochow University, Suzhou 215006, China

\section{Corresponding Author}

E-mail: ylsong@hit.edu.cn (Y. S). E-mail: jcxiaoicas@163.com (J.X). 
Table S1. Photophysical and electrochemical properties of PyPF, PyPT, PyPSe, and PyPTe.

\begin{tabular}{|c|c|c|c|c|}
\hline & PyPF & PyPT & PyPSe & PyPTe \\
\hline $\mathrm{Abs}^{\text {a) }}[\mathrm{nm}]$ & $\begin{array}{l}337,354, \quad 387, \\
407\end{array}$ & $\begin{array}{l}343,358,402, \\
423\end{array}$ & $\begin{array}{l}342,358,402, \\
424\end{array}$ & $\begin{array}{l}348,367,407, \\
430\end{array}$ \\
\hline $\mathrm{PL}^{\text {a) }}[\mathrm{nm}]$ & 447,470 & 459,486 & 478 & - \\
\hline$\left.\Phi F^{a}\right)$ & 0.68 & 0.54 & 0.08 & - \\
\hline$\tau^{\text {a) }}[\mathrm{ns}]$ & 9.18 & 7.22 & - & - \\
\hline $\left.\mathrm{HOMO}^{\mathrm{b}}\right)[\mathrm{eV}]$ & -5.27 & $-5.13^{\mathrm{e})}$ & $-5.12^{\mathrm{e})}$ & -5.09 \\
\hline $\mathrm{HOMO}^{\mathrm{c})}[\mathrm{eV}]$ & -5.29 & -5.26 & -5.22 & -5.15 \\
\hline $\mathrm{LUMO}^{\mathrm{c})}[\mathrm{eV}]$ & -1.95 & -1.97 & -1.96 & -1.94 \\
\hline Band gap ${ }^{\mathrm{d}}[\mathrm{eV}]$ & 3.38 & 3.34 & 3.32 & 3.30 \\
\hline
\end{tabular}

a) In toluene; b)Derived from the cyclic voltammetry data in $\mathrm{CH}_{2} \mathrm{Cl}_{2}$; c) Obtained from DFT calculation; d) Obtained from TD-DFT calculation; e) Ref 1

Table S2. Values of energies, oscillator strengths and dominant contributions of the respective molecular orbitals for $\mathrm{S}_{0} \rightarrow \mathrm{S}_{1}$ of PyPF, PyPT, PyPSe, and PyPTe.

\begin{tabular}{lccc}
\hline & Energy (eV) & Oscillator strengths & Major MO $\rightarrow$ MO contributions (\%) \\
\hline PyPF & 3.38 & 0.2099 & LUMO $\rightarrow$ HOMO (91\%) \\
PyPT & 3.34 & 0.2030 & LUMO $\rightarrow$ HOMO (92\%) \\
PyPSe & 3.32 & 0.2012 & LUMO $\rightarrow$ HOMO (92\%) \\
PyPTe & 3.30 & 0.1986 & LUMO $\rightarrow$ HOMO (90\%) \\
\hline
\end{tabular}


(a)

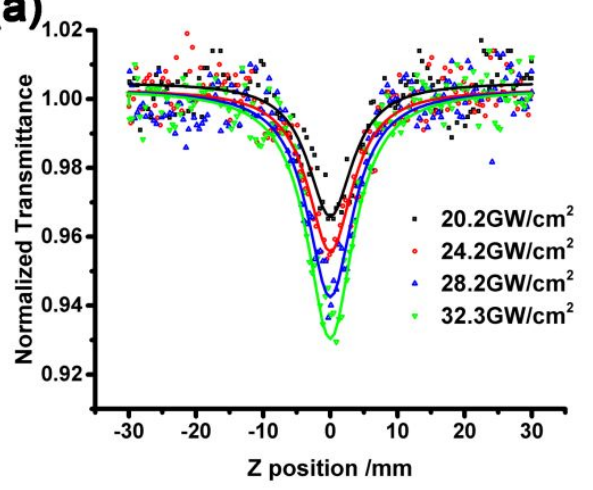

(c)

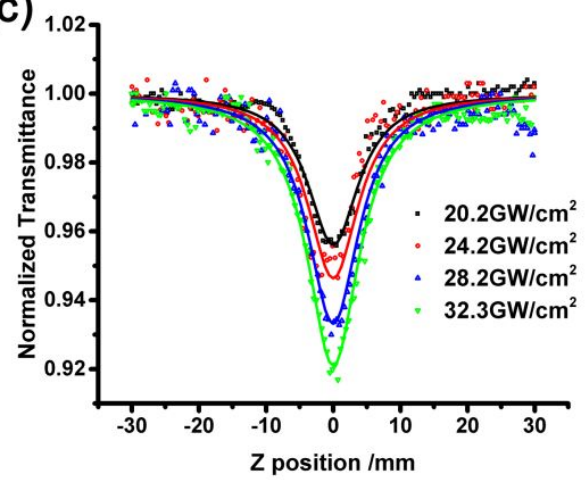

(e)

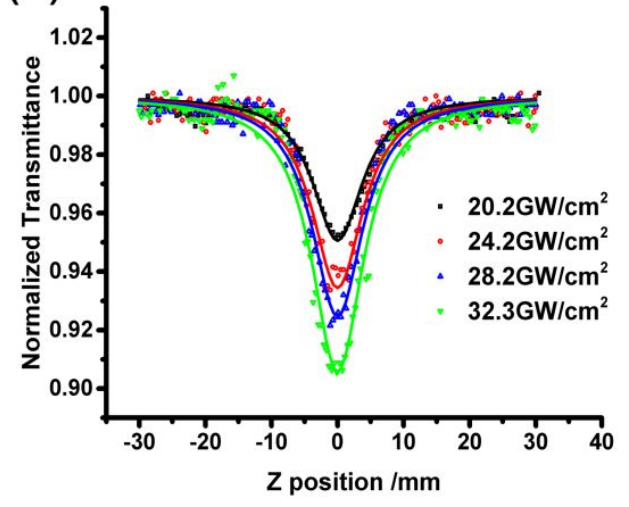

(g)

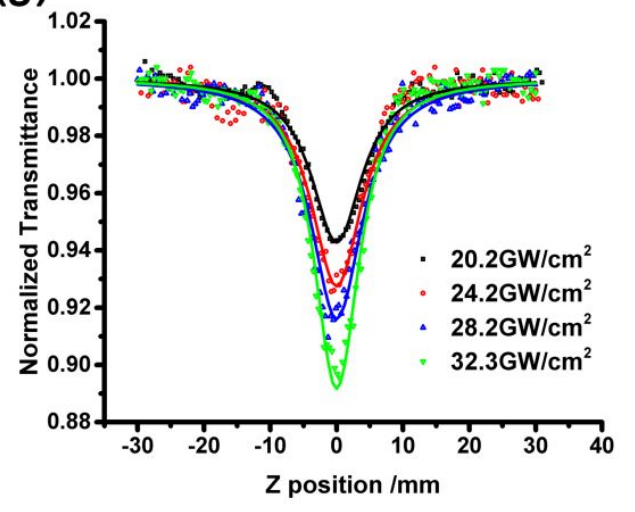

(b)

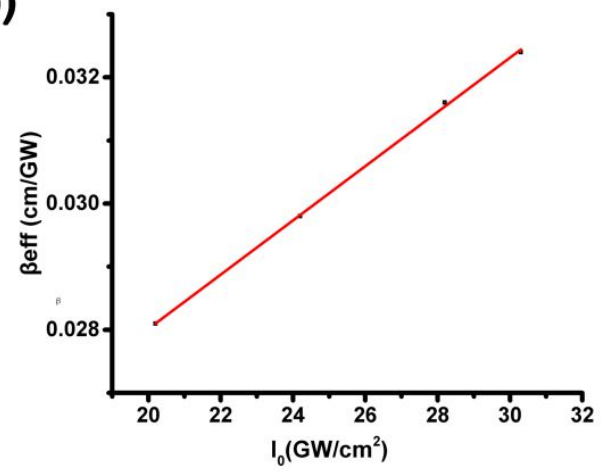

(d)

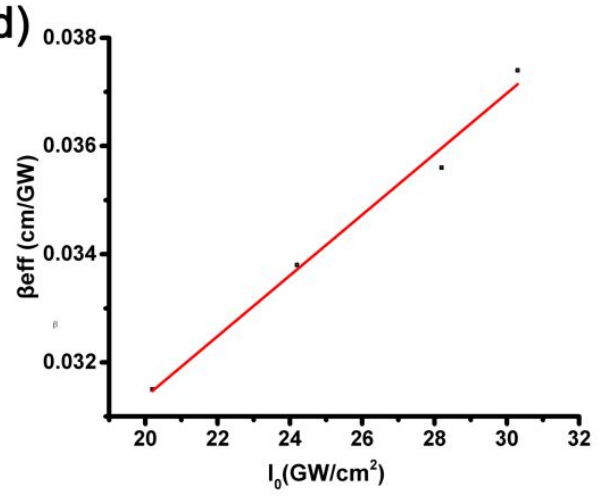

(f)

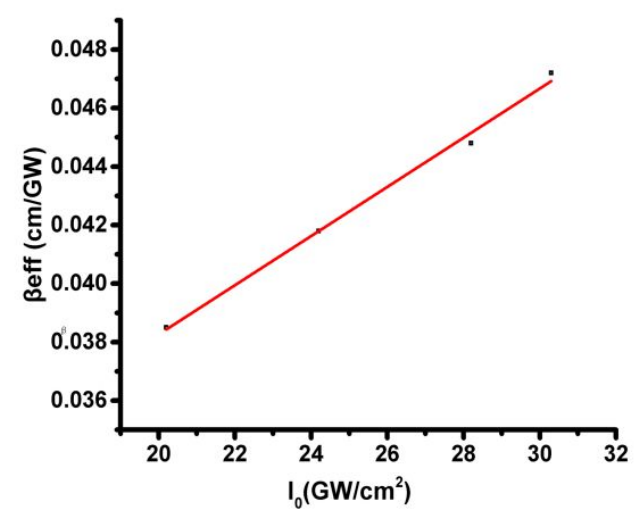

(h)

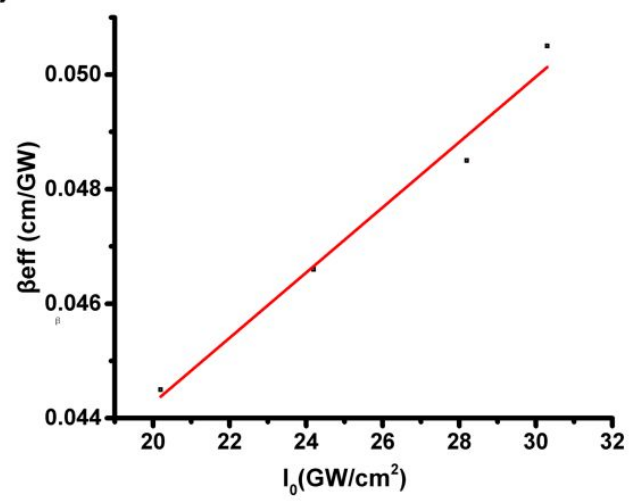


Figure S1. Open-aperture Z-scan data at different energies under $532 \mathrm{~nm}$ with a $190 \mathrm{fs}$ pulse for PyPF (a), PyPT (c), PyPSe (e), PyPTe (g). The dots are the experimental data and the corresponding solid lines represent the numerical ftting. The input laser intensity dependence of nonlinear absorption coefficient are for PyPF (b), PyPT (d), PyPSe (f), PyPTe (h).
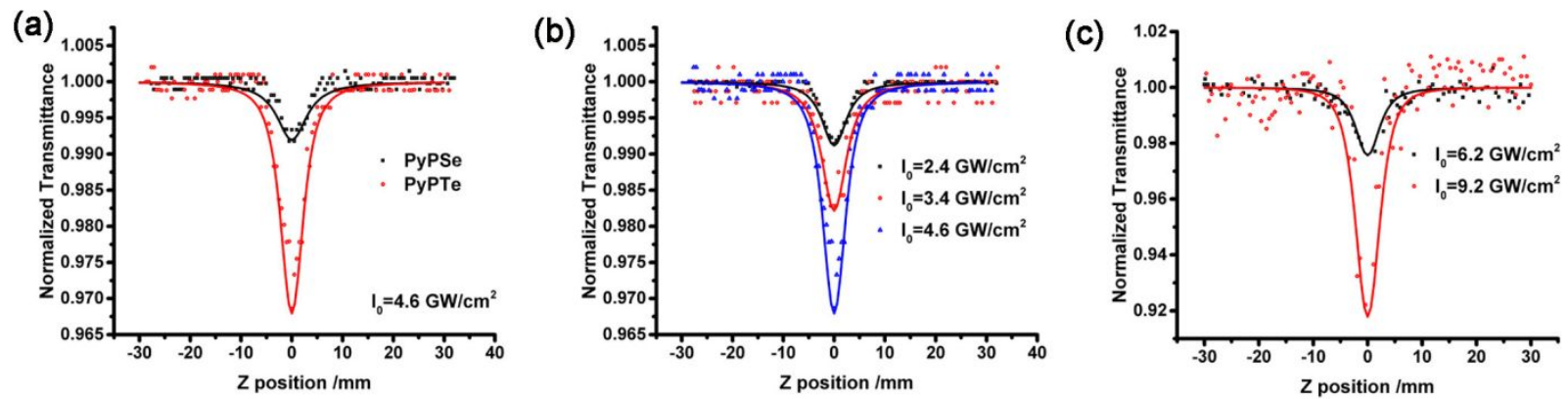

Figure S2. (a) Open-aperture Z-scan of PyPSe (black dots) and PyPTe (red dots) under $532 \mathrm{~nm}$ with a 21ps pulse. (b) Open-aperture Z-scan of PyPTe at different energies under $532 \mathrm{~nm}$ with a laser pulse 21 ps. (c) Open-aperture Z-scan of PyPTe with a laser pulse 4 ns. The dots are the experimental data and the corresponding solid lines represent the numerical ftting.

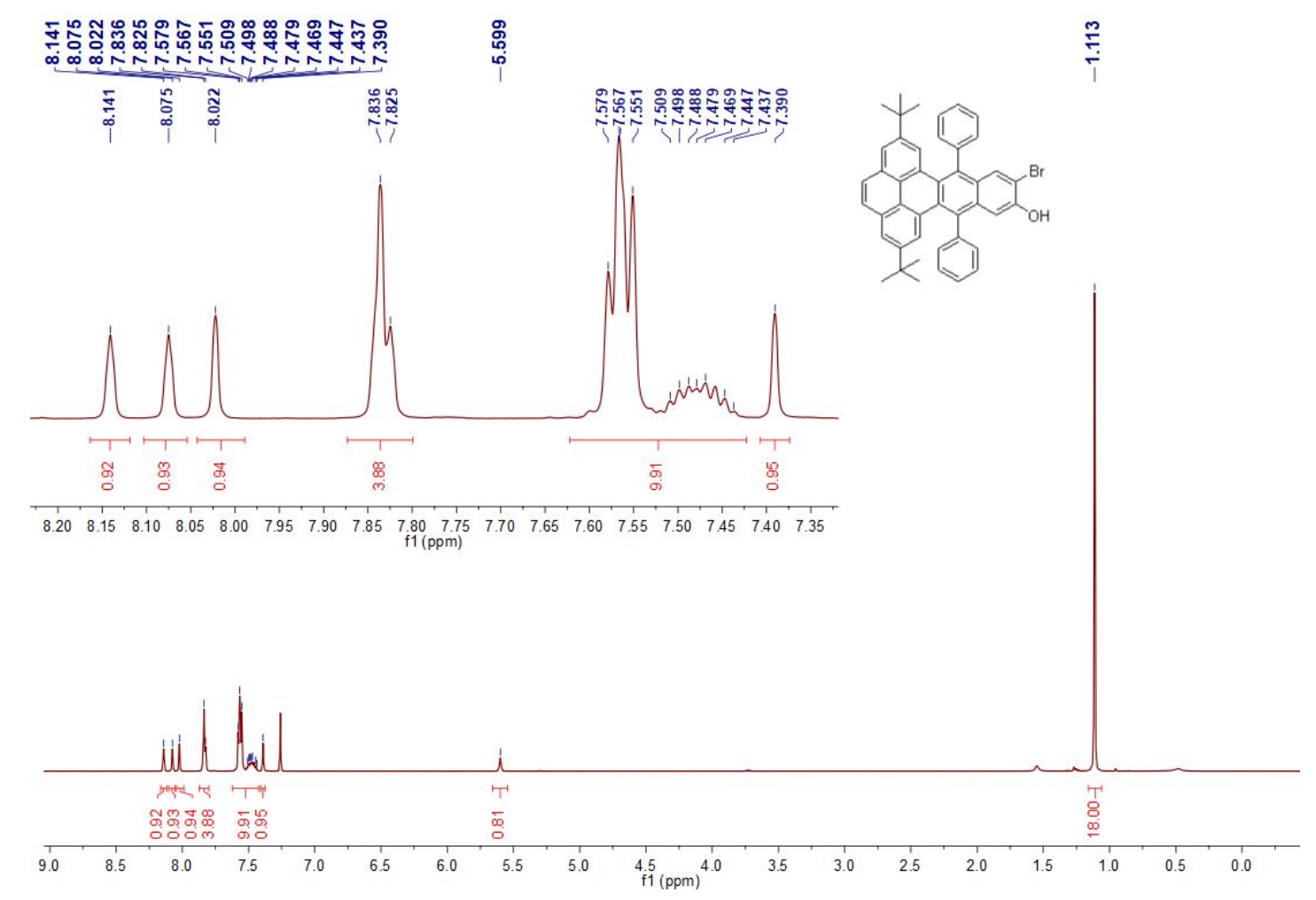


Figure S3. ${ }^{1} \mathrm{H}$ NMR spectrum of 2.

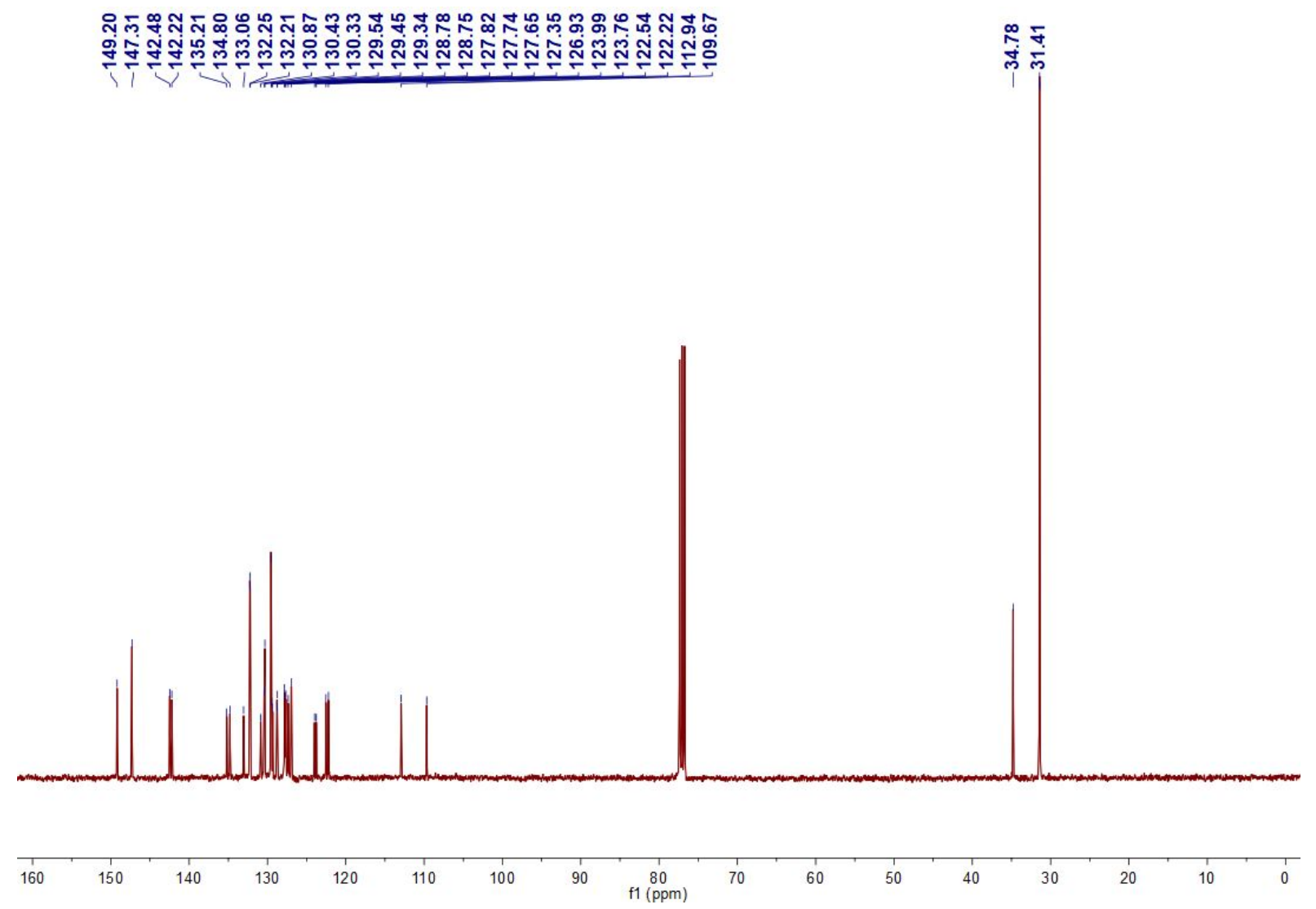

Figure S4. ${ }^{13} \mathrm{C}$ NMR spectrum of 2 . 


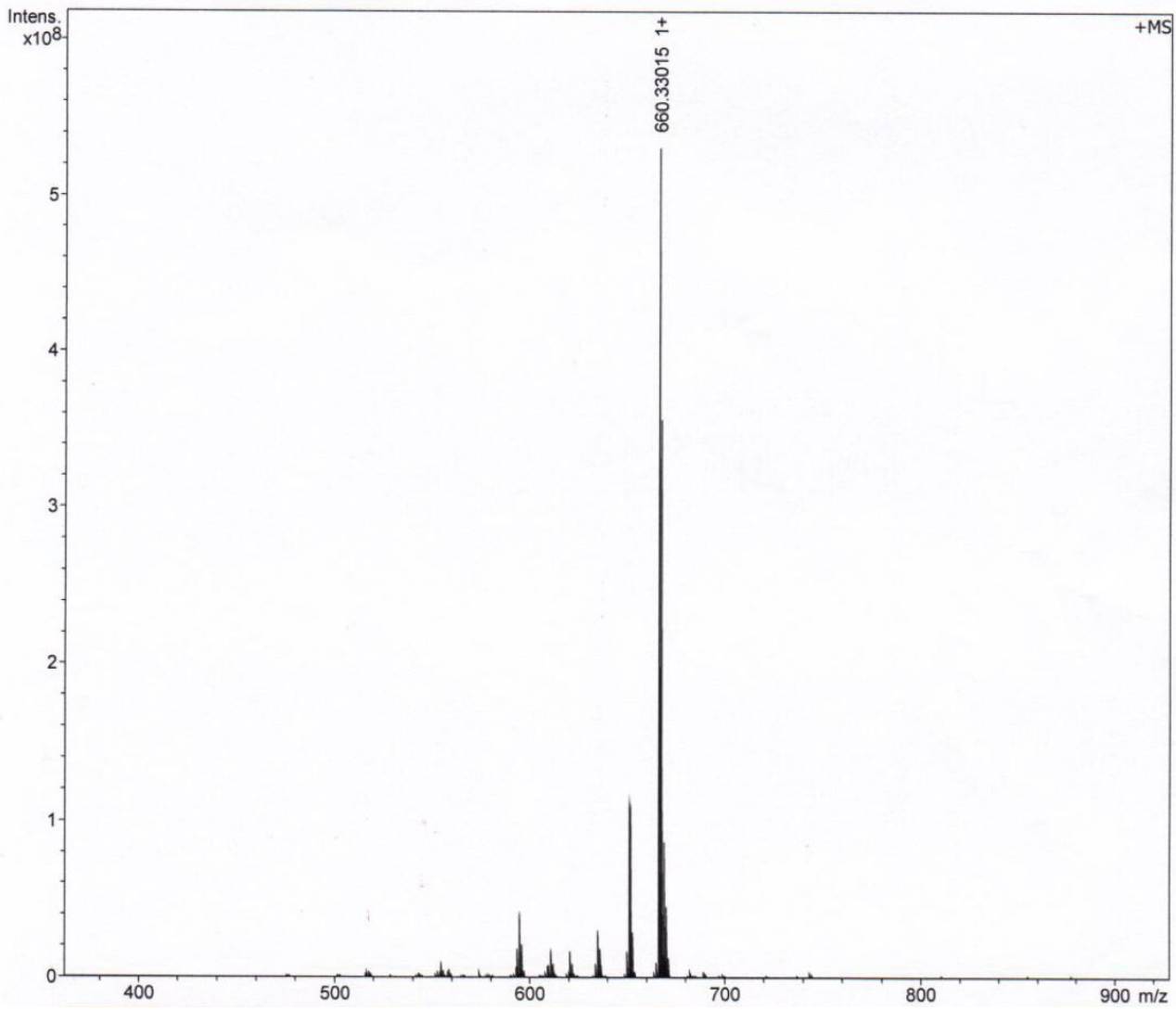

Figure S5. MALDI-TOF spectrum of 2. 


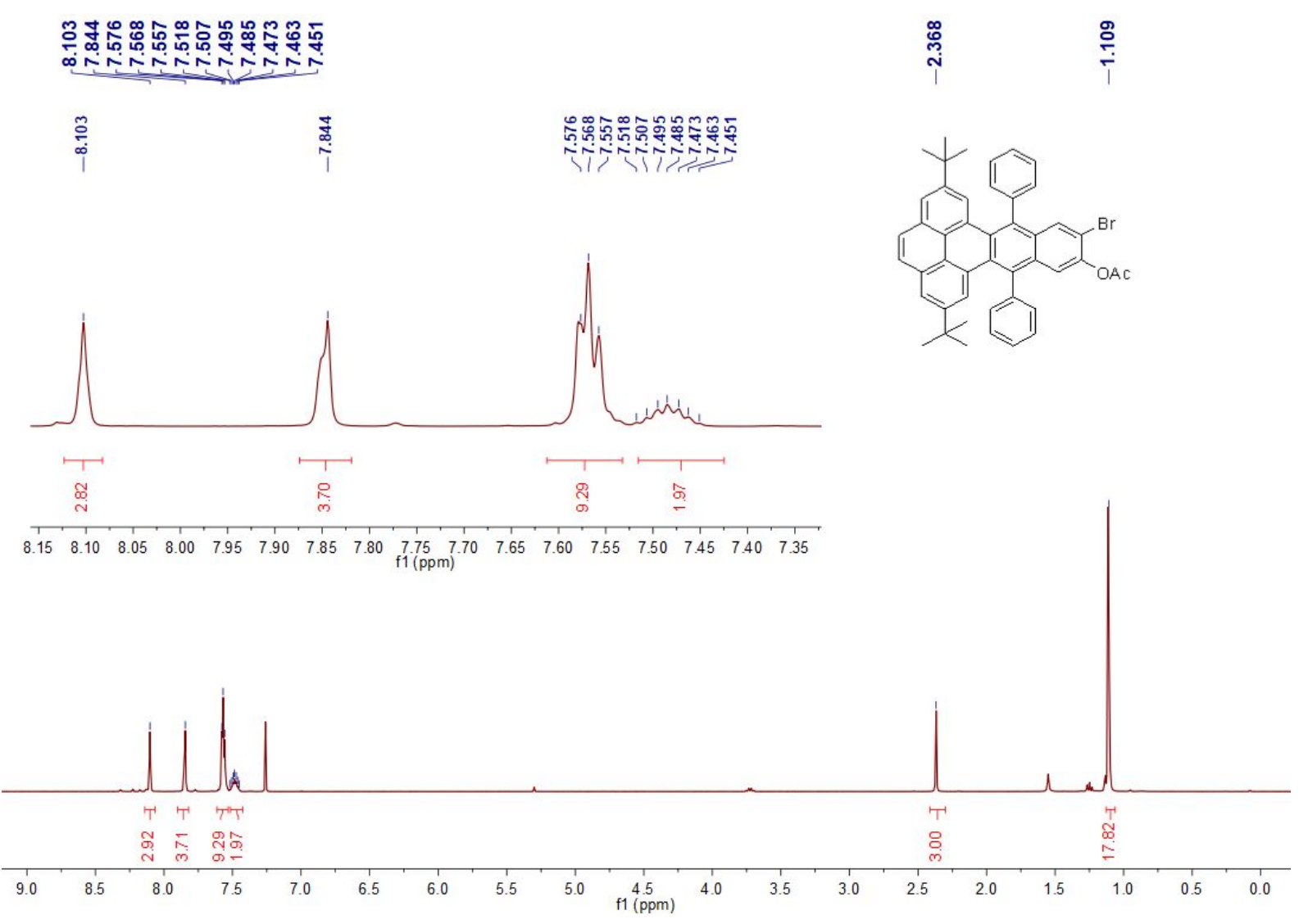

Figure S6. ${ }^{1} \mathrm{H}$ NMR spectrum of 3 . 


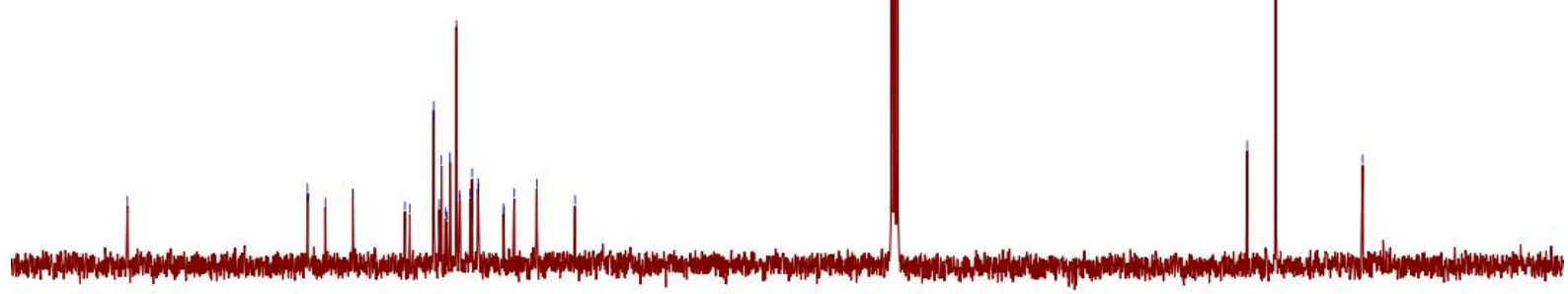

$150 \quad 140$

90
$1(\mathrm{ppm})$

80

Figure S7. ${ }^{13} \mathrm{C}$ NMR spectrum of $\mathbf{3}$. 


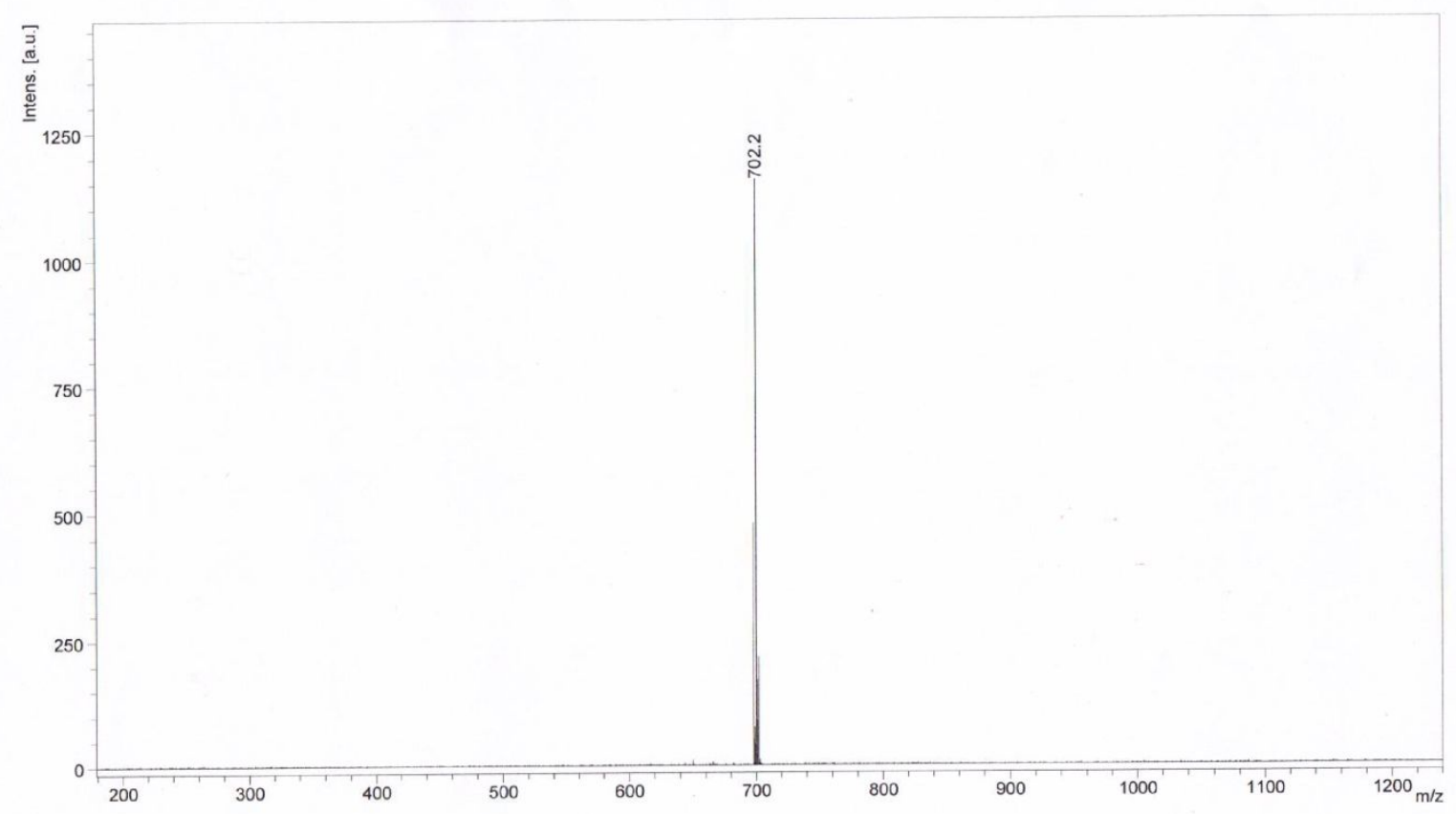

Figure S8. MALDI-TOF spectrum of 3.

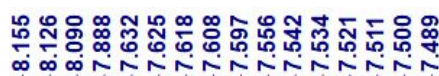

$\underset{0}{\infty}$

io

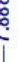

กำ

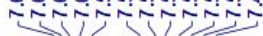

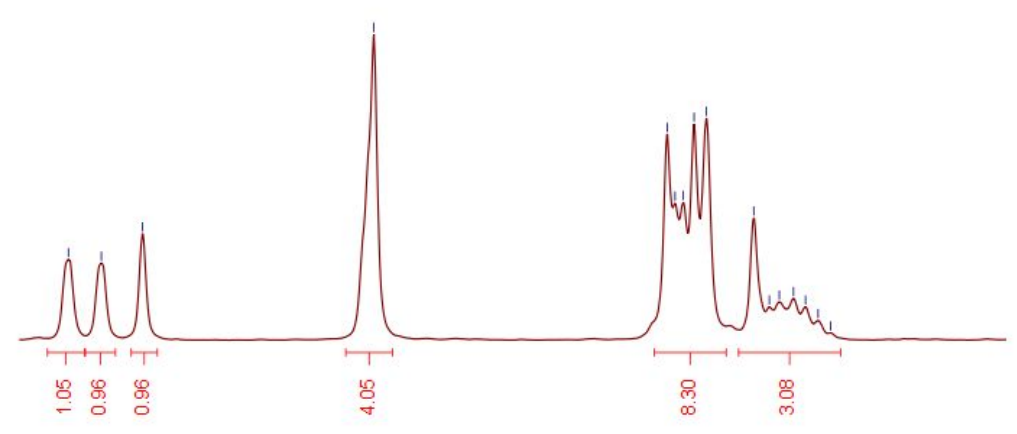

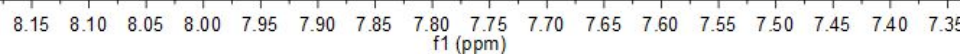
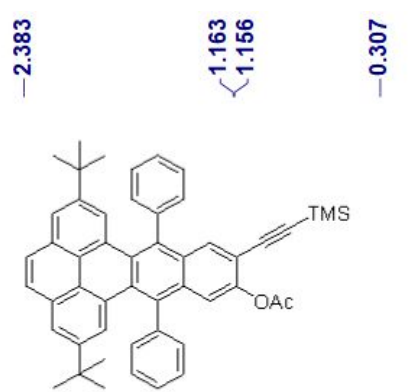

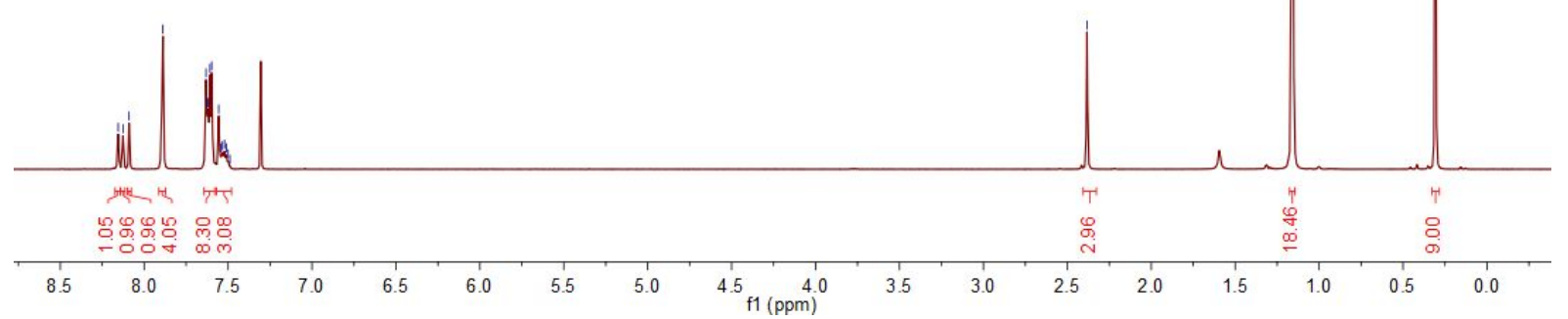


Figure S9. ${ }^{1} \mathrm{H}$ NMR spectrum of 4 .
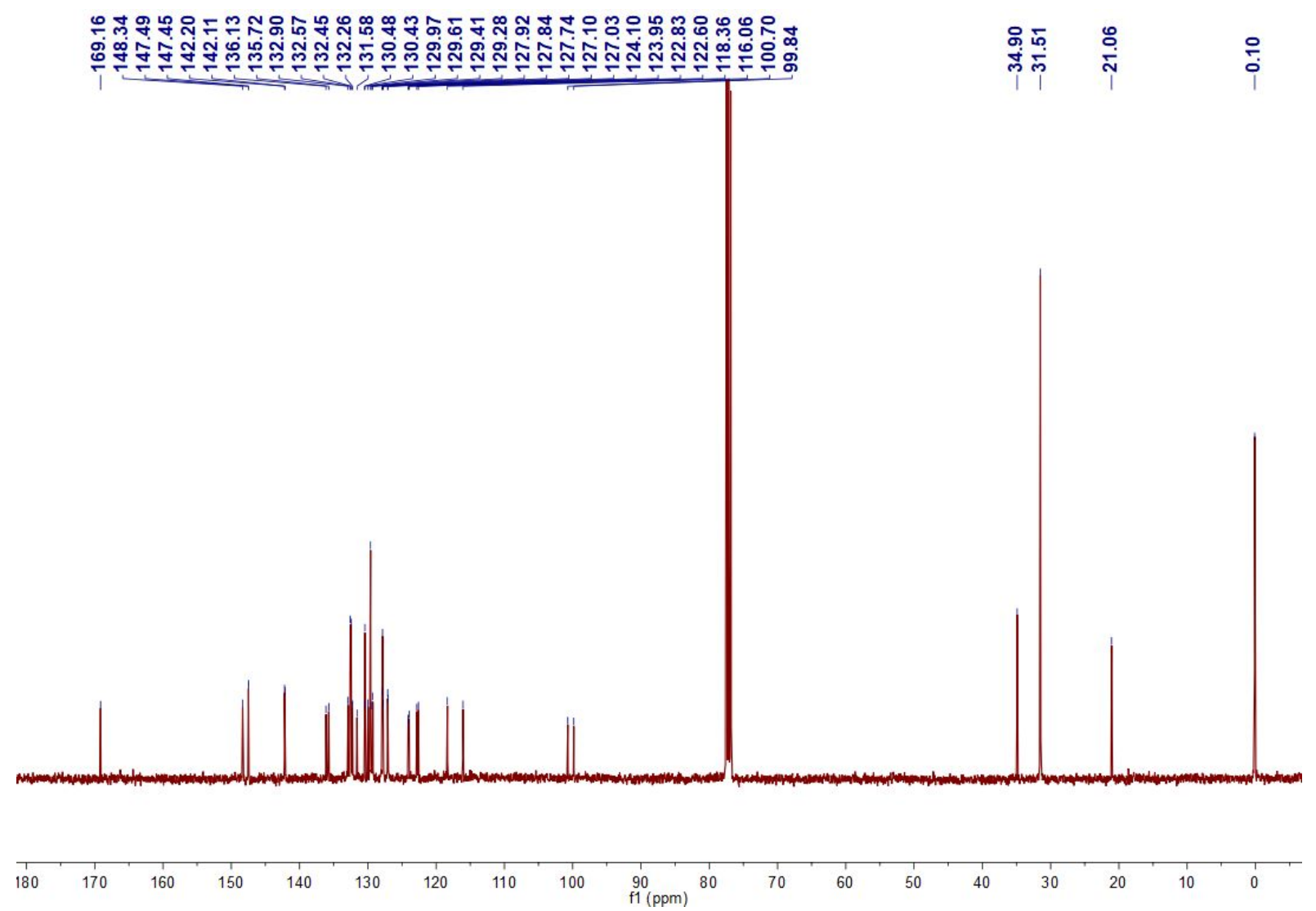

Figure S10. ${ }^{13} \mathrm{C}$ NMR spectrum of 4 .

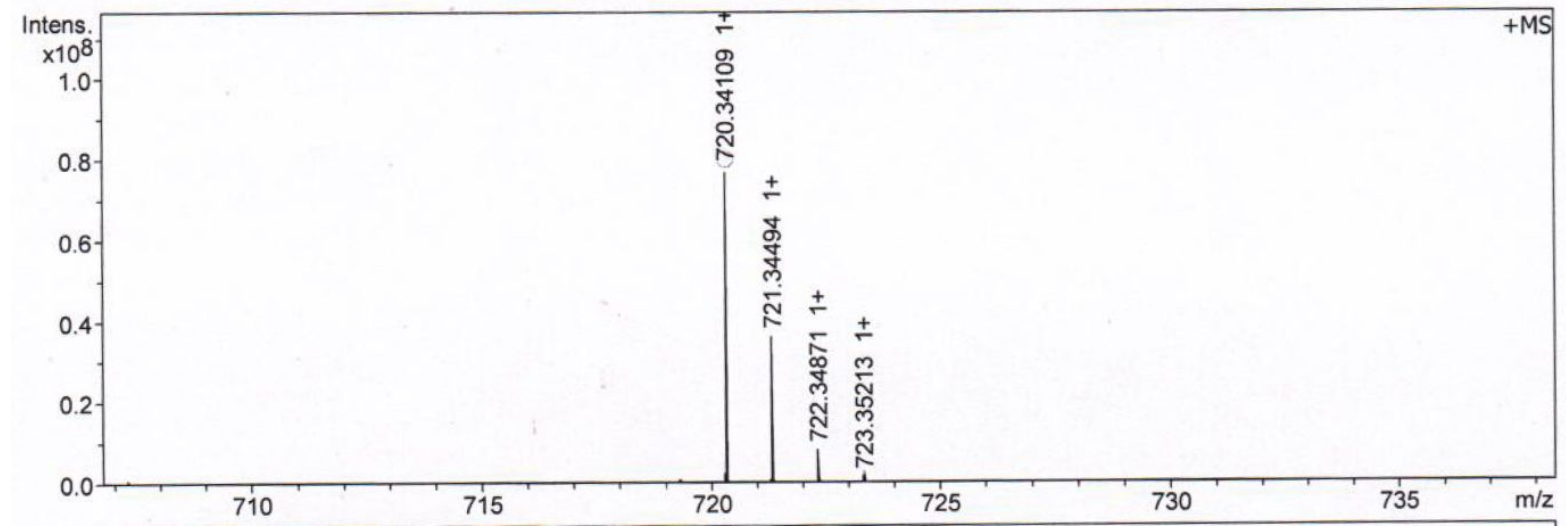

Figure S11. HR-MS spectrum of 4. 


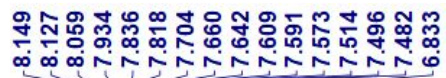

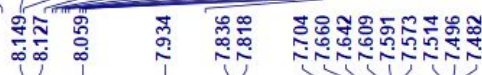

๘

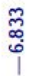

$\stackrel{\stackrel{2}{\leftarrow}}{i}$
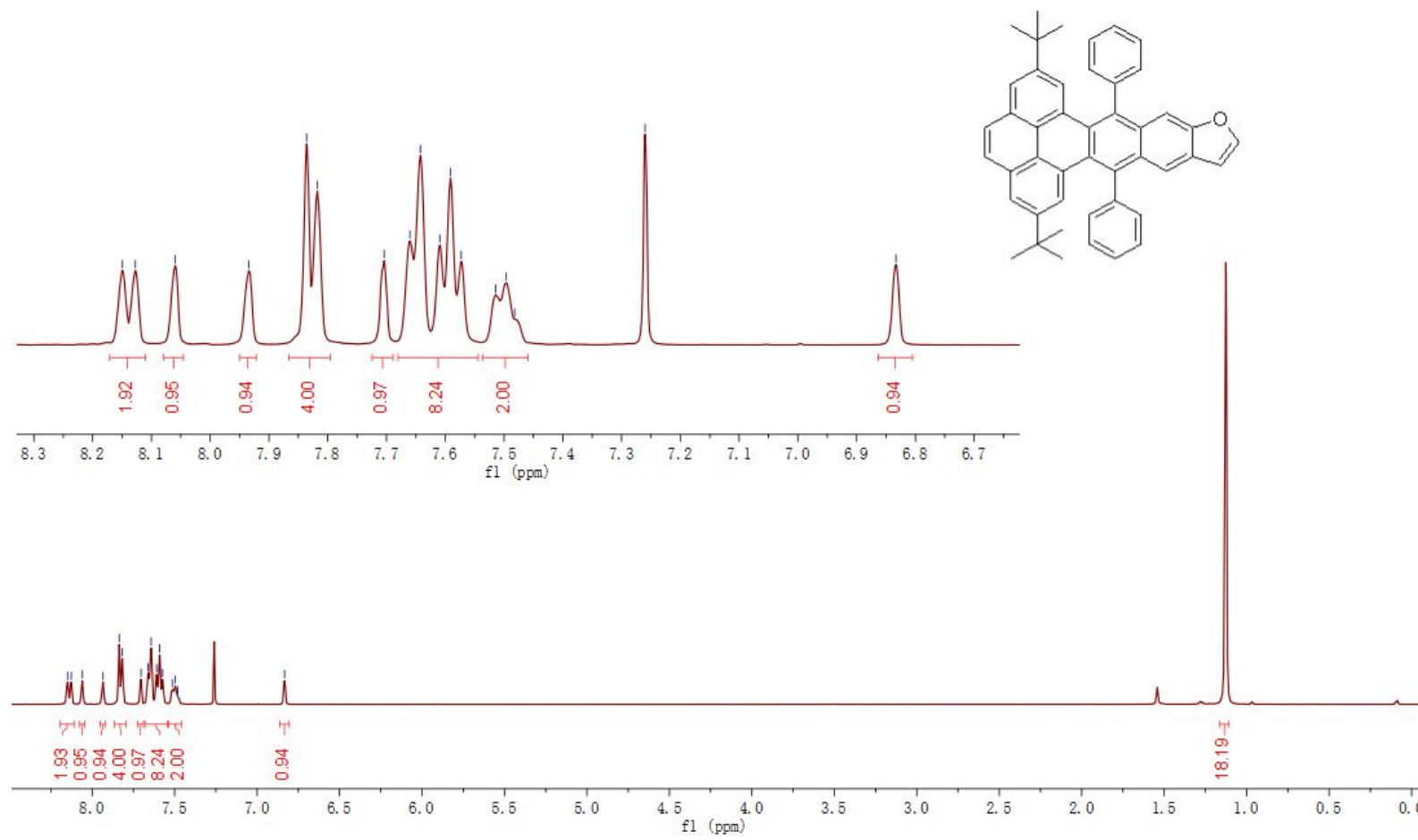

Figure S12. ${ }^{1} \mathrm{H}$ NMR spectrum of PyPF. 

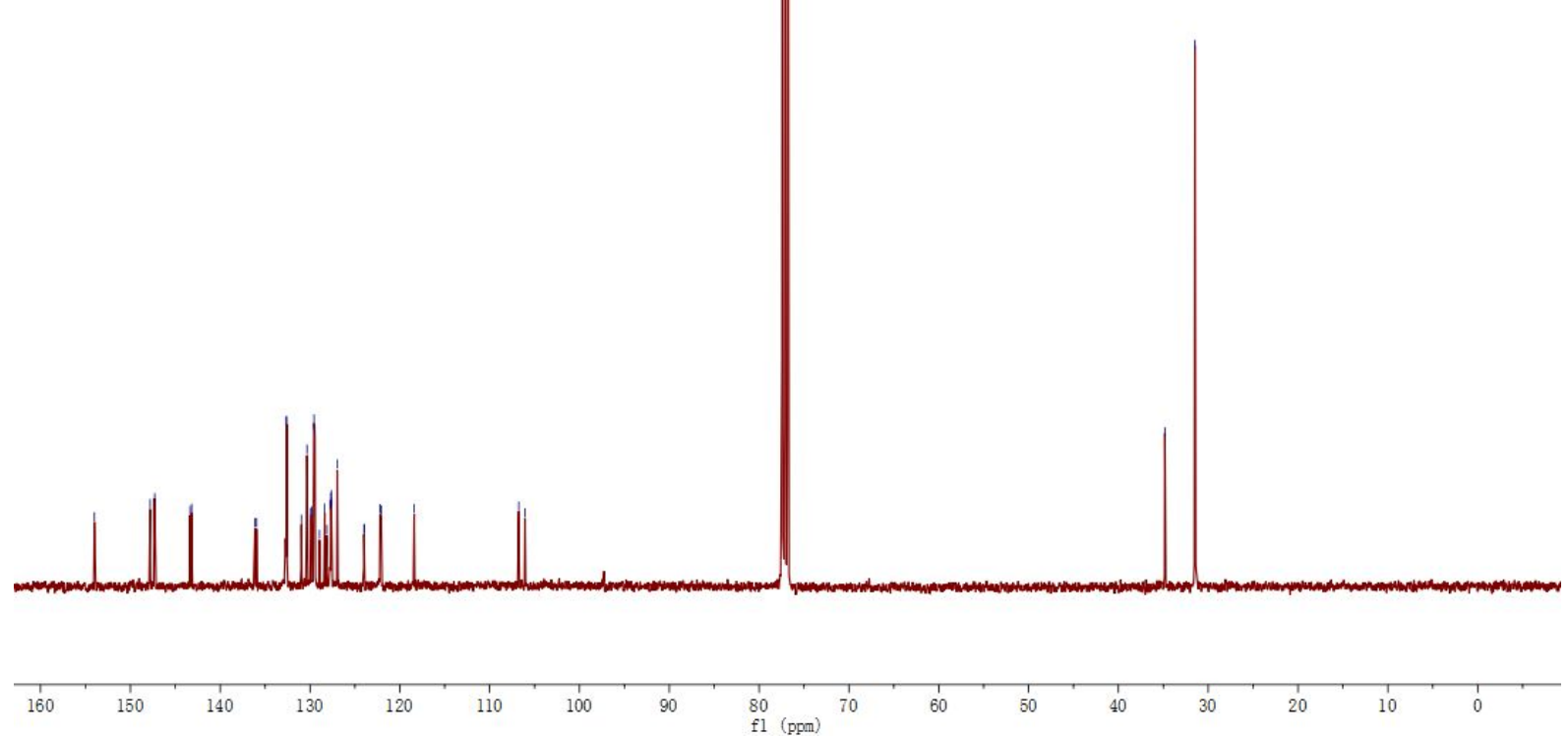

Figure S13. ${ }^{13} \mathrm{C}$ NMR spectrum of PyPF.

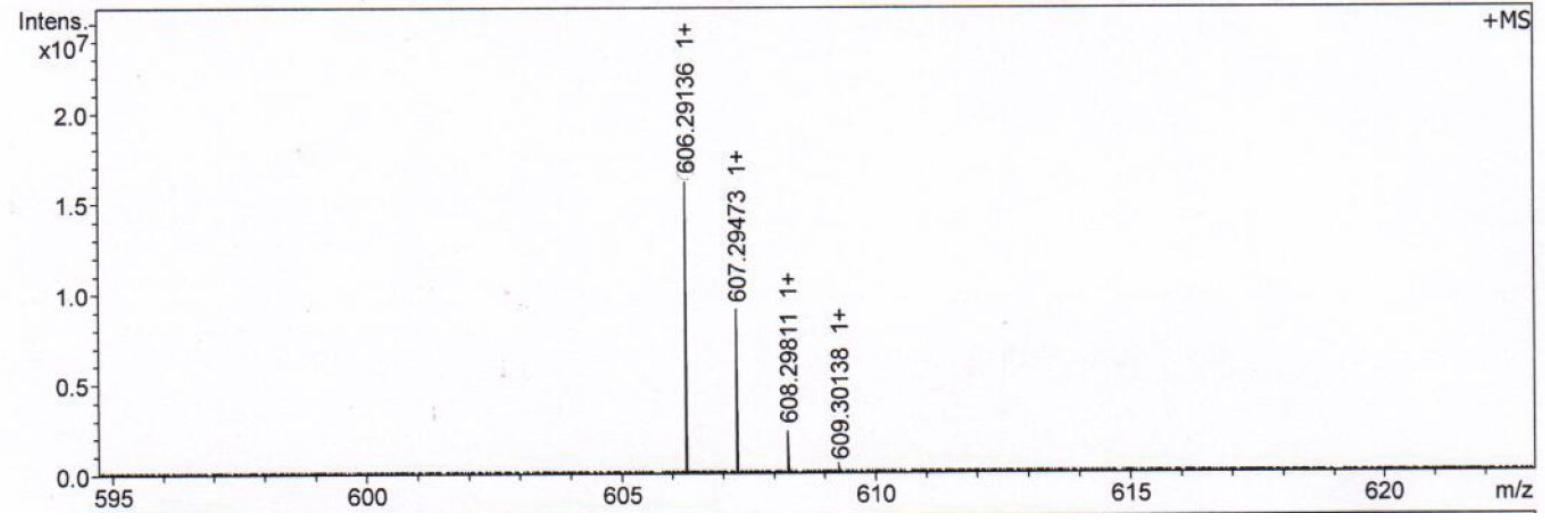

Figure S14. HR-MS spectrum of PyPF. 


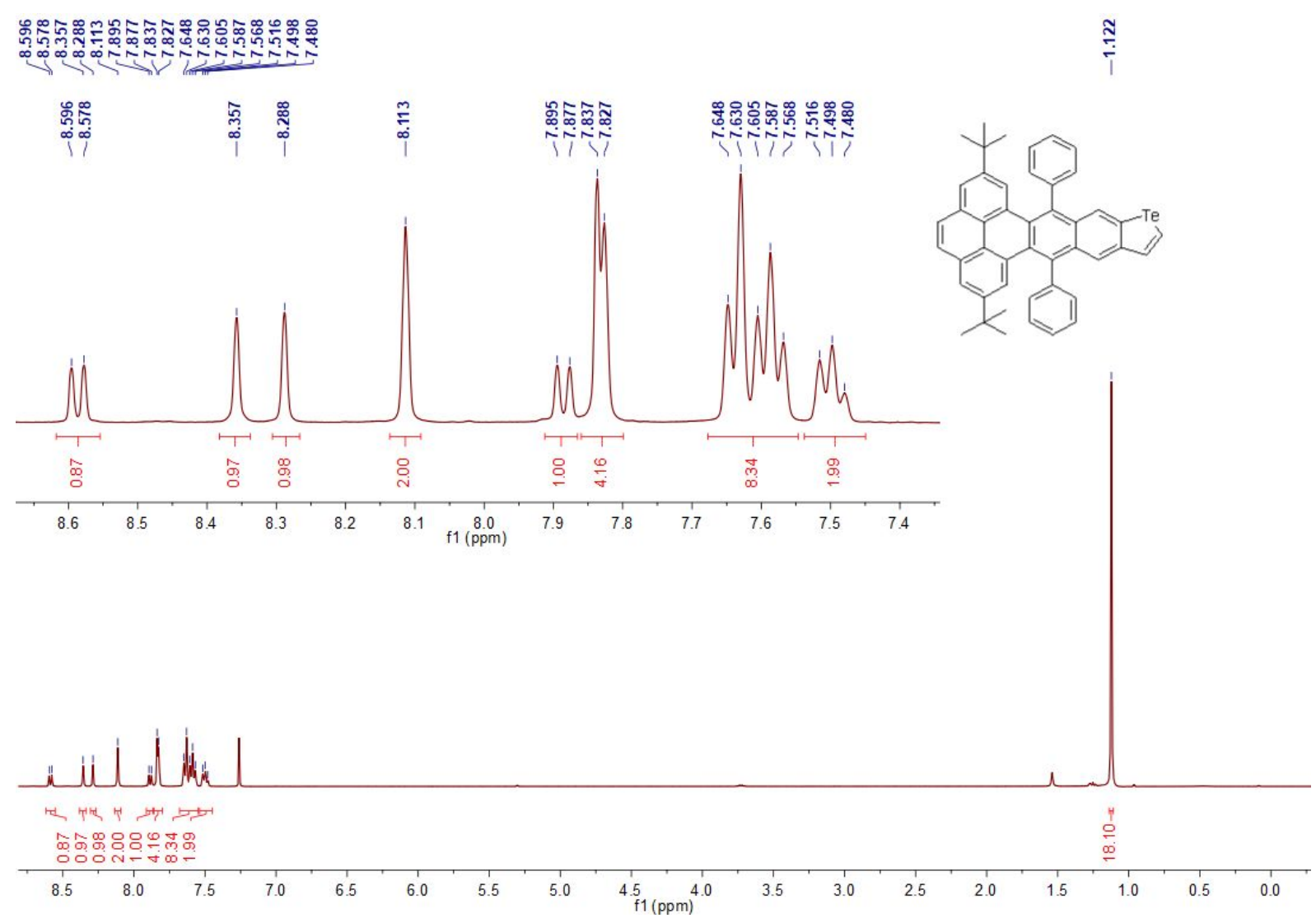

Figure $\mathbf{S 1 5}{ }^{1} \mathrm{H}$ NMR spectrum of 2 . 


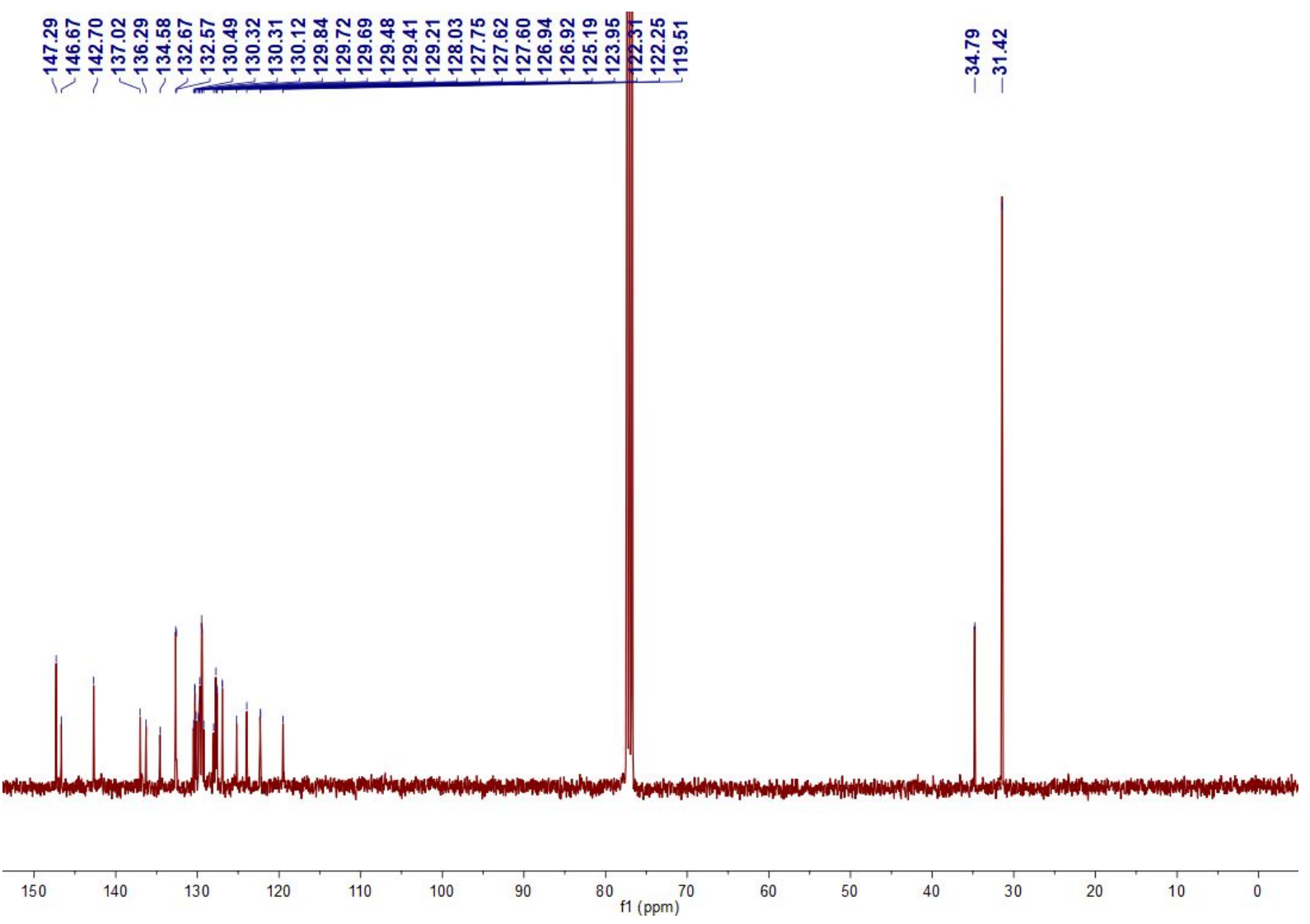

Figure S16. ${ }^{13} \mathrm{C}$ NMR spectrum of PyPTe.

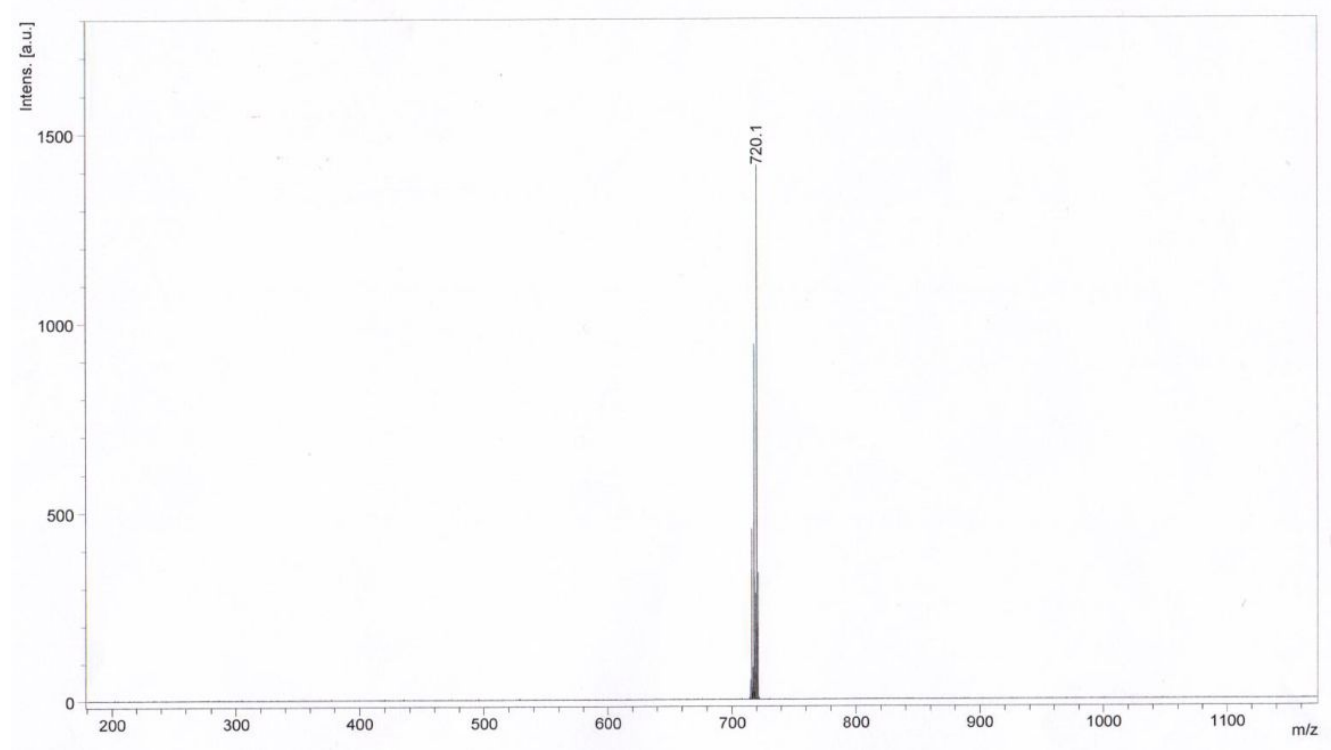

Figure S17. MALDI-TOF spectrum of PyPTe.

S14 


\section{Reference}

(1) Lv, B.; Shen, X.; Xiao, J.; Duan Lv, B.; Shen, X.; Xiao, J.; Duan, J.; Wang, X.; Yi, Y. Synthesis, Single Crystal, and Physical Properties of Asymmetrical Thiophene/SelenopheneFused Twistacenes. Chem.--Asian J. 2015, 10, 2677-2682. 